\title{
Resection of Vaginal Septum in Obstructed Hemivagina and Ipsilateral Renal Anomaly: Utility of Three Dimensional Intraoperative Ultrasound
}

\author{
Conway Xu' ${ }^{1}$, Shannon M. Osborne ${ }^{1}$, Jonia Alshiek ${ }^{1}$, S. Abbas Shobeiri ${ }^{1}$, Rachel K. Casey ${ }^{1,2^{*}}$ \\ ${ }^{1}$ Department of Obstetrics and Gynecology, Inova Fairfax Hospital, Falls Church, Virginia, USA \\ ${ }^{2}$ Pediatric Specialists of Virginia, Fairfax, Virginia, USA \\ Email: *rkcasey@gmail.com
}

How to cite this paper: $\mathrm{Xu}, \mathrm{C} .$, Osborne, S.M., Alshiek, J., Shobeiri, S.A. and Casey, R.K. (2021) Resection of Vaginal Septum in Obstructed Hemivagina and Ipsilateral Renal Anomaly: Utility of Three Dimensional Intraoperative Ultrasound. Open Journal of Obstetrics and Gynecology, 11, 355-359. https://doi.org/10.4236/ojog.2021.114035

Received: February 16, 2021

Accepted: March 12, 2021

Published: March 15, 2021

Copyright $\odot 2021$ by author(s) and Scientific Research Publishing Inc. This work is licensed under the Creative Commons Attribution International License (CC BY 4.0).

http://creativecommons.org/licenses/by/4.0/

\begin{abstract}
Background: Obstructed hemivagina and ipsilateral renal anomaly (OHVIRA) syndrome is a rare congenital condition of the female urogenital tract, presenting intraoperative challenges. We demonstrate the utility of three dimensional intraoperative ultrasound to better delineate anatomy and aid in optimal resection of the vaginal septum. Case: A 12-year-old female was referred to pediatric and adolescent gynecology for irregular periods and evaluation of her gynecologic organs. Imaging studies confirmed OHVIRA syndrome. She underwent uncomplicated vaginal septum resection, guided by three dimensional intraoperative ultrasound. Summary and Conclusion: Intraoperative ultrasound can better delineate challenging anatomy. We show that three dimensional intraoperative ultrasound offers significant advantages over traditional two dimensional ultrasound and is a supplement to MRI, which may be helpful in complex anatomical cases like OHVIRA syndrome.
\end{abstract}

\section{Keywords}

OHVIRA Syndrome, 3D Intraoperative Ultrasound

\section{Introduction}

Obstructed hemivagina and ipsilateral renal anomaly (OHVIRA) syndrome is a rare congenital condition of the female urogenital tract. It was first reported in 1922 and since then, it has garnered increasing attention which has contributed to a better understanding of the heterogeneity behind the underlying anatomy 
[1]. This can include uterine didelphys or, more rarely, septate uterus or single uterus, along with renal anomalies such as ipsilateral renal agenesis, renal duplication, multicystic dysplastic kidney, ureteral duplication, ectopic ureter, or rectovesical bands [1] [2] [3] [4]. Despite the varying anatomy, patients with OHVIRA syndrome usually have the typical presentation of a postmenarchal female with dysmenorrhea or pelvic pain, oftentimes cyclical and worsening over time. On examination, there is a visible vaginal sidewall bulge along with an unobstructed cervix. Further imaging such as pelvic ultrasound and magnetic resonance imaging (MRI) usually confirms obstructed hemivagina and uterine didelphys [5] [6]. Management options traditionally include diagnostic laparoscopy for better definition of anatomy and treatment of concurrent adhesions or endometriosis and vaginoplasty to alleviate the obstructed hemivagina. At times, a two stage vaginoplasty may be necessary to better delineate tissue planes when there is significant anatomic distortion from hematocolpos and chronic inflammation [1]. However, over time, single stage vaginoplasty has become preferred and is usually sufficient for alleviation of the obstructed hematocolpos and the patient's symptoms.

While there is now a better understanding of the heterogeneity in the underlying anatomy of OHVIRA, there can still be challenges behind safe and minimally invasive surgical intervention. In this patient, we demonstrate the utility of three dimensional intraoperative ultrasound to better delineate the complete dimensions of the obstructed hemivagina and aid in optimal resection of the vaginal septum.

\section{Case}

A 12-year-old female was referred to pediatric and adolescent gynecology for irregular periods and evaluation of her gynecologic organs. She had menarche about 1 year prior to presentation and her periods were monthly, but would often come a few days early or late. At the time of her initial consultation, she had already been following with pediatric nephrology for a known solitary left kidney since birth. Her medical history was otherwise insignificant and non-contributory. She underwent a pelvic ultrasound which showed a Mullerian duct uterine anomaly with two endometrial cavities and two probable cervical canals along with mild distension in the vaginal canal. A follow up MRI confirmed uterine didelphys with obstructed right hemivagina (Figure 1(a) and Figure 1(b)). As such, the patient and her mother were counseled and she was scheduled for resection of her vaginal septum to alleviate her obstructed hemivagina.

Prior to excision of her vaginal septum, exam under anesthesia noted a slight bulge along the right vaginal sidewall (Figure 2(a)) and a palpable left cervix. As the vaginal septum was only slightly bulging and not typical of more common OHVIRA presentations with more significant hematocolpos, three dimensional intraoperative ultrasound was performed to better delineate the dimensions of the obstructed right hemivagina (Figure 3 ) and determine optimal limits of surgical 


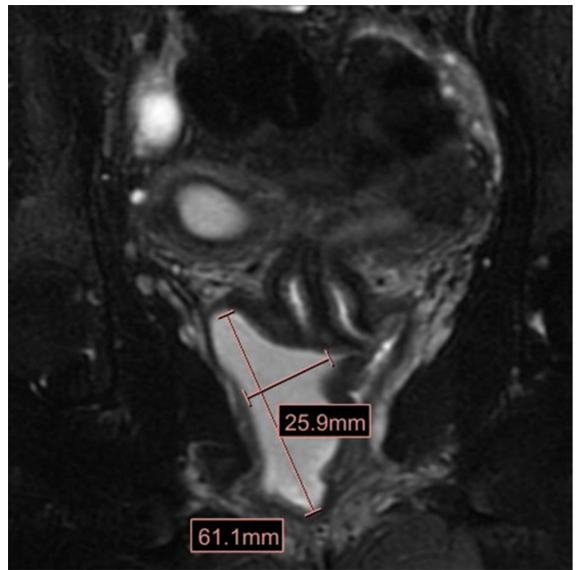

(a)

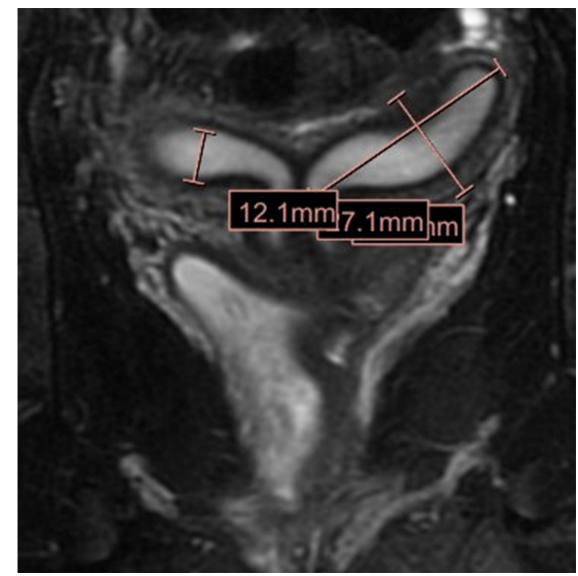

(b)

Figure 1. (a) MRI coronal view depicting obstructed right hemivagina; (b) MRI coronal view depicting uterine didelphys.

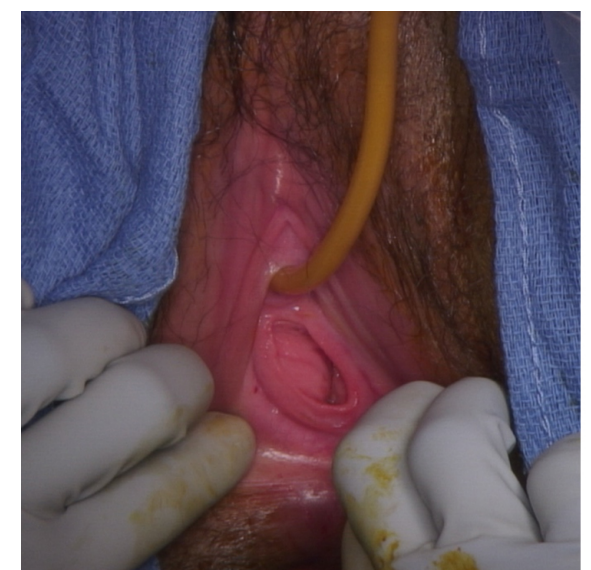

(a)

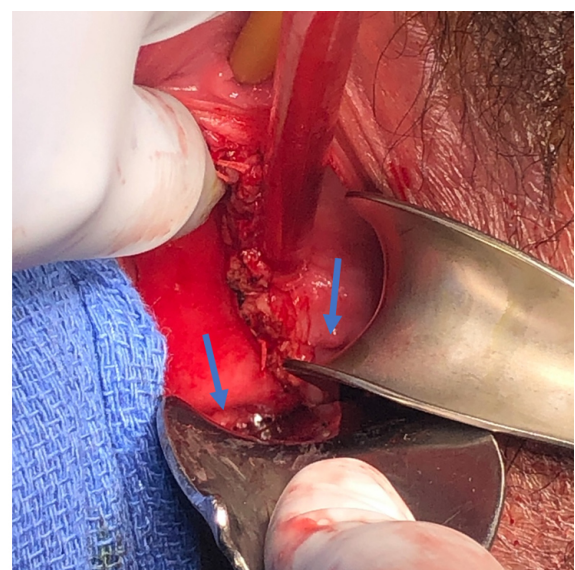

(b)

Figure 2. (a) Obstructed right hemivagina prior to excision; (b) After excision of vaginal septum, two separate cervices are visualized. Arrows at cervical os.

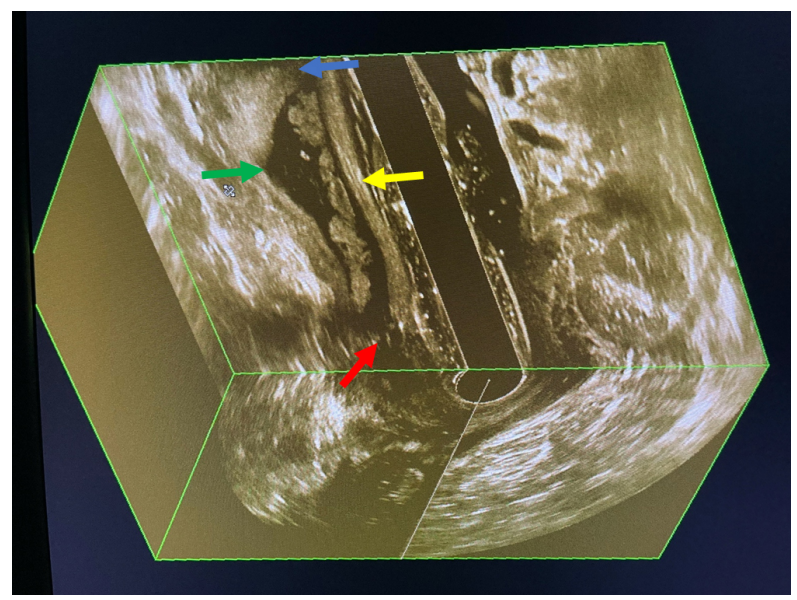

Figure 3. Three dimensional ultrasound of obstructed right hemivagina. Red Arrow (distal limit of obstruction), Blue Arrow (proximal limit of obstruction), Green Arrow (lateral limit of obstruction), Yellow Arrow (vaginal septum). 
resection. The septum was initially incised with electrocautery at the distal margin. There was light yellow mucus noted upon entry into the obstructed hemivagina. After further exploration, there was no evidence of old hematocolpos. Kelly clamps were then applied anteriorly and laterally against the vaginal walls. The remainder of the vaginal septum was then excised with electrocautery. The remaining pedicles were suture ligated for hemostasis. Afterwards, examination of the vagina was notable for two separate cervices (Figure 2(b)). The patient tolerated the procedure well and had an unremarkable post-operative course. The patient was seen in the office at 2 weeks and 2 months post-operatively. Her vaginal incisions were well healed and both of her cervixes were normal to palpation.

\section{Discussion}

The use of intraoperative ultrasound is not a novel technique and can be very helpful in better delineating challenging anatomy. In fact, intraoperative ultrasound has been used in previous cases of OHVIRA syndrome [7] [8]. However, we demonstrate the first use of three dimensional intraoperative ultrasound, to our knowledge, in the management of OHVIRA syndrome.

In our case, the patient's vaginal septum was only slightly bulging, unlike the more typical presentation in OHVIRA patients, making the determination of optimal borders for surgical resection of her septum more difficult. Prior cases describing OHVIRA syndrome demonstrate the utility of more advanced imaging such as MRI to help identify more subtle findings such as hemivagina which may otherwise be missed on standard two dimensional ultrasound [6]. However, MRI is significantly limited in its ability to provide dynamic and immediate results in the operating room.

Using three dimensional intraoperative ultrasound in this case allows for better real time delineation of the complete dimensions of the obstructed hemivagina, allowing for more optimal surgical resection of the vaginal septum while avoiding surrounding critical structures. The ability to create a three dimensional profile offers a significant advantage in better understanding spatial relationships compared to traditional two dimensional ultrasound, especially when operating in close spaces such as the vagina.

\section{Conclusion}

Intraoperative ultrasound can better delineate challenging anatomy, especially within narrow spaces. We show that three dimensional intraoperative ultrasound offers significant advantages over traditional two dimensional ultrasound and is a supplement to MRI, which may be helpful in complex anatomical cases like OHVIRA syndrome.

\section{Disclosure/Conflict of Interest}

The authors report no proprietary or commercial interest in any product men- 
tioned or concept discussed in this article.

\section{References}

[1] Smith, N.A. and Laufer, M.R. (2007) Obstructed Hemivagina and Ipsilateral Renal Anomaly (OHVIRA) Syndrome: Management and Follow-Up. Fertility and sterility, 87, 918-922. https://pubmed.ncbi.nlm.nih.gov/17320871/

https://doi.org/10.1016/j.fertnstert.2006.11.015

[2] Han, B., Herndon, C.N., Rosen, M.P., Wang, Z.J. and Daldrup-Link, H. (2010) Uterine Didelphys Associated with Obstructed Hemivagina and Ipsilateral Renal Anomaly (OHVIRA) Syndrome. Radiology Case Reports, 5, 327.

https://pubmed.ncbi.nlm.nih.gov/27307842/ https://doi.org/10.2484/rcr.v5i1.327

[3] Shah, D.K. and Laufer, M.R. (2011) Obstructed Hemivagina and Ipsilateral Renal Anomaly (OHVIRA) Syndrome with a Single Uterus. Fertility and Sterility, 96, e39e41. https://pubmed.ncbi.nlm.nih.gov/21605855/ https://doi.org/10.1016/j.fertnstert.2011.05.013

[4] Schlomer, B., Rodriguez, E. and Baskin, L. (2015) Obstructed Hemivagina and Ipsilateral Renal Agenesis (OHVIRA) Syndrome Should Be Redefined as Ipsilateral Renal Anomalies: Cases of Symptomatic Atrophic and Dysplastic Kidney with Ectopic Ureter to Obstructed Hemivagina. Journal of Pediatric Urology, 11, 77-e1-6. https://pubmed.ncbi.nlm.nih.gov/25797857/ https://doi.org/10.1016/j.jpurol.2014.12.004

[5] Mandava, A., Prabhakar, R.R. and Smitha, S. (2012) OHVIRA Syndrome (Obstructed Hemivagina and Ipsilateral Renal Anomaly) with Uterus Didelphys, an Unusual Presentation. Journal of Pediatric and Adolescent Gynecology, 25, e23-e25. https://pubmed.ncbi.nlm.nih.gov/22421561/ https://doi.org/10.1016/j.jpag.2011.11.004

[6] Bajaj, S.K., Misra, R., Thukral, B.B. and Gupta, R. (2012) OHVIRA: Uterus Didelphys, Blind Hemivagina and Ipsilateral Renal Agenesis: Advantage MRI. Journal of Human Reproductive Sciences, 5, 67-70. https://pubmed.ncbi.nlm.nih.gov/22870020/ https://doi.org/10.4103/0974-1208.97811

[7] Breech, L.L. and Laufer, M.R. (2009) Müllerian Anomalies. Obstetrics and Gynecology Clinics of North America, 36, 47-68.

https://pubmed.ncbi.nlm.nih.gov/19344847/ https://doi.org/10.1016/j.ogc.2009.02.002

[8] Miller, R.J. and Breech, L.L. (2008) Surgical Correction of Vaginal Anomalies. Clinical Obstetrics and Gynecology, 51, 223-236. https://pubmed.ncbi.nlm.nih.gov/18463454/ https://doi.org/10.1097/GRF.0b013e31816d2181 